\title{
Cholecystokinin Activates a Variety of Intracellular Signal Transduction Mechanisms in Rodent Pancreatic Acinar Cells
}

\author{
John A. Williams, M. Dolors Sans, Mitsuo Tashiro, Claus Schäfer, M. Julia Bragado and Andrzej Dabrowski
}

Department of Physiology, University of Michigan, Ann Arbor, MI 48109, U.S.A.

\begin{abstract}
Cholecystokinin (CCK) acting through its $\mathrm{G}$ protein-coupled receptor is now known to activate a variety of intracellular signaling mechanisms and thereby regulate a complex array of cellular functions in pancreatic acinar cells. The best studied mechanism is the coupling through heterotrimeric $\mathrm{G}$ proteins of the $\mathrm{G}_{\mathrm{q}}$ family to activate a phospholipase $\mathrm{C}$ leading to an increase in inositol trisphosphate and release of intracellular $\mathrm{Ca}^{2+}$. This pathway along with protein kinase $\mathrm{C}$ activation in response to the increase in diacylglycerol stimulates the secretion of digestive enzymes by the process of exocytosis. CCK also activates signaling pathways in acini more related to other processes. The three mitogen activated protein kinase cascades leading to ERKs, JNKs and p38 MAPK are all activated by CCK. CCK activates the ERK cascade by PKC activation of Raf which in turn activates MEK and ERKs. JNKs are activated by a distinct mechanism whish requires higher concentrations of CCK. Both ERKs and JNKs are presumed to regulate gene expression. CCK activation of p38 MAPK also plays a role in regulating the actin cytoskeleton through phosphorylation of the small heat shock protein HSP27. The PI3K-PKB-mTOR pathway is activated by CCK and plays a major role in regulating protein synthesis at the translational level. This includes both activation of p70 S6K leading to phosphorylation of ribosomal protein S6 and the phosphorylation of the binding protein for initiation factor 4E leading to formation of the mRNA cap binding complex. Other signaling pathways activated by CCK receptors include NF- $\mathrm{KB}$ and a variety of tyrosine kinases. Further work is needed to understand how CCK receptors activate most of the above pathways and to better understand the biological events regulated by these diverse signaling pathways.
\end{abstract}

One of the most well studied target cells used to study receptors and signaling mechanism for cholecystokinin (CCK) is the rodent pancreatic acinar cell. These cells possess relatively large numbers of CCKA receptors and a readily measured biologic endpoint, the secretion of digestive enzymes such as amylase. The pancreas can be digested with collagenase to yield the functional unit, the isolated acinus (Williams et al. 1978), or with further digestion, isolated acinar cells. Isolated rat and mouse acini are very sensitive to CCK and have been used to study ligand-receptor interaction, measurement of classical second messengers, activation of protein kinases and various biological effects including secretion, protein synthesis and membrane transport (Williams \& Blevins 1993). They can also be used as a sensitive bioassay for plasma CCK (Liddle et al. 1984).

\section{Signaling mechanisms regulating enzyme secretion}

Most of the early work on isolated pancreatic cells, acini and lobules focused on secretion of digestive enzymes and rapidly identified an increase in intracellular $\mathrm{Ca}^{2+}$ as the major controller by which CCK and the neurotransmitter, acetylcholine activated enzyme secretion (Yule \& Williams 1994). Acinar cells were instrumental in identifying the im-

Author for correspondence: John A. Williams, Department of Physiology, University of Michigan, 7744 Medical Science II, Ann Arbor, MI 48109-0622, USA (fax +1 734936 8813, e-mail jawillms@umich.edu). portance of intracellular $\mathrm{Ca}^{2+}$ release and later the identification of inositol trisphosphate (IP3) as the mediator of $\mathrm{Ca}^{2+}$ release. This early work led to a model which has been continued to be filled in whereby the liganded CCK receptor activates heterotrimeric $G$ proteins of the $G_{q}$ family, especially $G_{q}$ and $G_{11}$. The $G$ protein subunits then dissociate and the $\alpha$ subunit activates a phospholipase C- $\beta$ which cleaves phosphatidylinositol 4,5-bisphosphate to form IP3 and diacylglycerol (Matozaki \& Williams 1989). IP3 binds to an intracellular receptor (the IP3R) which functions as a ligand gated ion channel to release sequestered intracellular $\mathrm{Ca}^{2+}$ and diacylglycerol activates protein kinase C. High concentrations of CCK also activate $\mathrm{G}_{\mathrm{s}}$ and thereby adenylate cyclase leading to an increase in cAMP. Details on this research have been reviewed previously (Yule \& Williams 1994; Williams 2001).

Considerable work has also been carried out localizing the sites of $\mathrm{Ca}^{2+}$ release in the apical pole of the acinar cell and describing patterns of intracellular $\mathrm{Ca}^{2+}$ signaling (Petersen et al. 1999). Moreover, recent work has established the existence of alternative $\mathrm{Ca}^{2+}$ mobilizing messengers including cyclic ADP ribose and nicotinic acid adenine dinucleotide phosphate (NAADP) (Williams $2001 \& 2002$ ). The end result is that $\mathrm{Ca}^{2+}$ and protein kinase $\mathrm{C}$ can be shown together to mediate the secretion of digestive enzymes by exocytosis of zymogen granule content. As for other membrane fusion events, evidence indicates the participation of SNARE proteins and small $\mathrm{G}$ proteins, particularly Rab3D (Chen et al. 2002). An interaction with ac- 
tin present in a subluminal membrane network that granules must traverse is also involved. How $\mathrm{Ca}^{2+}$ triggers exocytosis, however, is still not clear. A number of kinases and phosphatases are activated by $\mathrm{Ca}^{2+}$, some are substrate specific such as myosin light chain kinase and others are more multifunctional such as $\mathrm{Ca}^{2+}$-calmodulin activated kinase II and protein kinase C. However, it is not clear whether any of these actions trigger secretion or whether there is a $\mathrm{Ca}^{2+}$ sensor on the granule membrane that activates exocytosis after docking and priming steps which involve phosphorylation events.

\section{Non-secretory signaling pathways activated by CCK}

Acinar cells are also regulated in terms of growth, gene expression, translational control of protein synthesis, cytoskeletal organization and energy metabolism. Although the classical second messengers such as $\mathrm{Ca}^{2+}$, diacylglycerol, and cAMP may regulate these functions, a number of novel signaling pathways are also involved. Current research is identifying these pathways in acinar cells, showing regulation by CCK and illuminating the relationship of the pathway to specific cellular functions. In most cases less is known of how the CCKA receptor activates the pathway. In some cases this may be through a different heterotrimeric $\mathrm{G}$ protein such as $\mathrm{G}_{12 / 13}$. In others it involves small $\mathrm{G}$ proteins such as Ras, Rac and Rho. It is still not clear whether all of these novel pathways are activated directly by proteinprotein interactions or indirectly by $\mathrm{Ca}^{2+}$ and other classi- cal second messengers. In some cases non-receptor tyrosine kinases may also be involved.

\section{Mitogen-activated kinase cascades.}

Mitogen-activated protein kinases (MAPKs) are serinethreonine directed kinases that were originally identified as mitogen-activated but are now known to be activatable by a number of hormones, neurotransmitters and different kinds of stress. MAPKs are known to regulate a number of cellular processes and to be involved in control of cell growth, differentiation, survival, apoptosis and cytokine production. All MAPK cascades are composed of three protein kinases acting in series (Widmann et al. 1999). Thus each MAPK is activated by a MAPK kinase (MAPKK or MKK) which is in turn activated by a MAPK kinase kinase (MAPKKK or MKKK). Each MAPK is activated by dual phosphorylation of tyrosine and threonine residues in a $\mathrm{T}$ $\mathrm{X}-\mathrm{Y}$ sequence and phosphospecific antibodies to these epitopes are now often used to assess activation of each pathway. At the upstream end of each cascade MAPKKKs receive information through small $\mathrm{G}$ proteins of the Ras superfamily. Currently, there are five independent MAPK cascades. Three have been identified in pancreatic acinar cells and all are activated by CCK and CCKA receptors. These are the cascades leading to ERKs, JNKs and p38 and are diagrammed in fig. 1.

CCK was first shown to rapidly increase tyrosine phosphorylation and kinase activity of ERK1/2, originally known as p44 and p42 MAPK respectively based on their

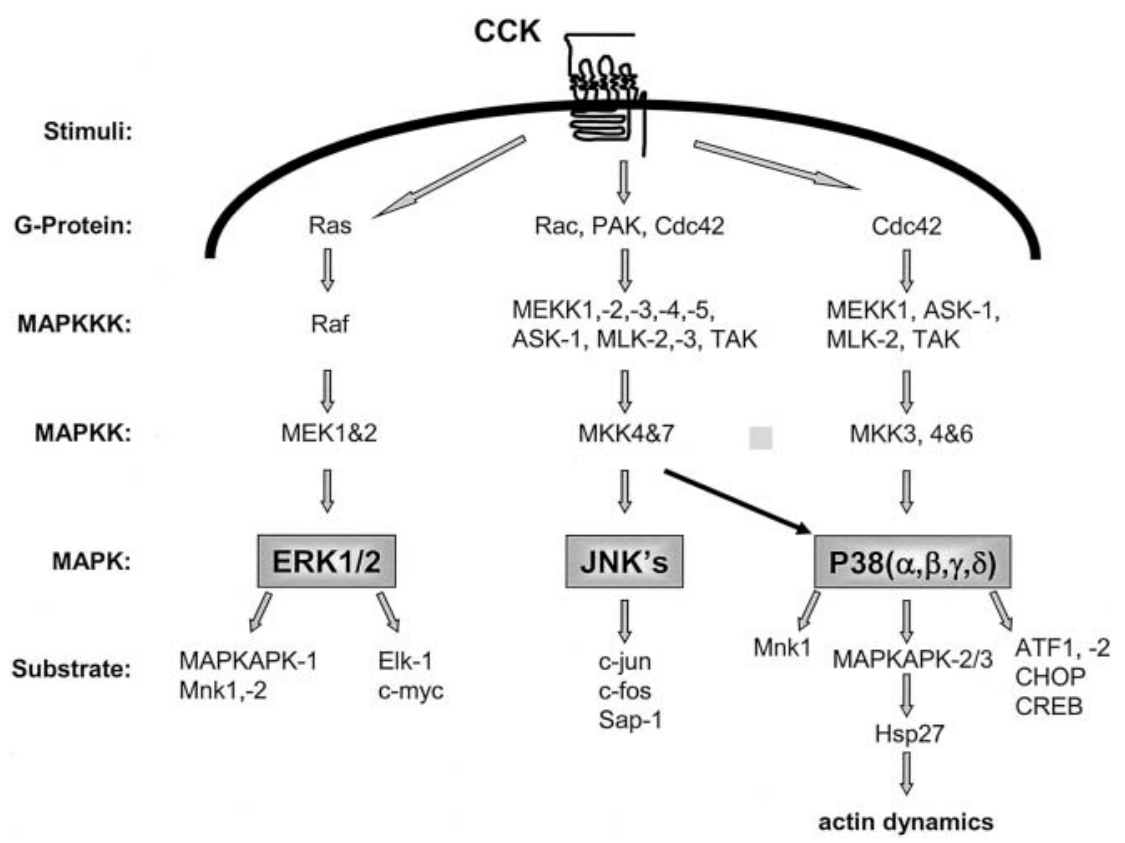

Fig. 1. Schematic dipiction of the three mitogen-activated protein kinase (MAPK) signaling cascades known to be activated by CCK receptors. Each three-module kinase cascade is shown being activated by a small G protein. Individual MAPK's signal to the nucleus by phosphorylating transcription factors either directly or through other kinases. In addition, p38 has demonstrated effects on actin dynamics. While CCK activates ERKs, JNKs and p38 in pancreatic acinar cells, specific upstream components activated by CCK in the JNK and p38 cascades remain to be identified and the multiplicity of potential regulators are taken from a variety of cell types. Activation of these cascades coordinates the regulation of different genetic programs. Modified from Schäfer \& Williams (2000). 
molecular mass (Duan \& Williams 1994). Subsequently, CCK was shown to increase the activity of the upstream kinases MEK1/2 (Duan et al. 1995) three forms of Raf (RafA RafB and cRaf1) which are the MKKK for this pathway (Dabrowski et al. 1997). A kinase downstream of ERK, p90 RSK also known as MAPKAPK-1 was also shown to be activated by CCK (Bragado et al. 1997). Most of these assays involve immunoprecipitation of specific proteins followed by a kinase assay. Two MEK inhibitors, PD98059 and U0126 block ERK and p90RSK activation in acinar cells. Both ERKs and p90RSK phosphorylate transcription factors such as Elk and c-myc as well as Histone $\mathrm{H} 3$.

Classically, the ERK cascade is activated by Ras but the importance of Ras in CCK activation of this MAPK cascade is unclear. Both CCKA and CCKB receptors activate ERKs in a transfected model cell (Dabrowski et al. 1997b) and gastrin activates ERKs in the pancreas derived AR42J cell line (Seva et al. 1997). Dabrowski et al. (1996a \& 1997a) found that CCK and EGF activate ERK1/2 by different mechanisms. EGF stimulation induced autophosphorylation of the EGF receptor and had a strong effect to increase tyrosine phosphorylation of the adapter protein Shc and formation of a Shc-Grb2-SOS complex where SOS is a known Ras guanine nucleotide exchange factor. EGF also increased the fraction of Ras which had GTP bound and is presumed to be the activated state which can bind Raf. These actions were unaffected by the protein kinase $\mathrm{C}$ antagonist GF-109203X. By contrast, CKK had no effect on EGF receptor phosphorylation. Some other $G$ protein coupled receptors are known to act by "transactivating" the EGF receptor but this does not seem to be the case for the CCKA receptor in rat acinar cells (Dabrowski et al. 1996a). CCK had a weaker effect on Shc phosphorylation, forma- tion of a Shc-Grb2-SOS complex and no measurable effect on Ras GTP. However, the actions of CCK were blocked by the protein kinase $\mathrm{C}$ inhibitor. Because $\mathrm{CCK}$ is a stronger activator of Raf than is EGF and this action is blocked by the protein kinase $\mathrm{C}$ inhibitor, it was postulated that $\mathrm{CCK}$ receptor leads to activation of the ERK kinase cascade by protein kinase $\mathrm{C}$ phosphorylation of Raf. These differences are diagrammed in fig. 2. Activation of Ras by CCK is unclear, however, as an earlier study showed, CCK increased the rate of GTP binding to Ras in permeabilized acini (Duan et al. 1995). The lack of importance of active Ras in the action of CCK to activate ERKs was also shown by Nicke et al. (1999) who used an adenoviral vector to express dominant negative N17 Ras in overnight cultured rat acini. Expression of this dominant negative Ras blocked the action of EGF to activate ERKs but had no effect on ERK activation by CCK. It did, however, inhibit DNA synthesis and blocked activation of JNKs. These results support the concept of different mechanisms for CCK and EGF to activate ERKs. It is possible that the tyrosine phosphorylation of Shc induced by CCK is involved in regulation a different pathway. The nature of the tyrosine kinase involved remains to be identified although it could well be a member of the Src family or $\mathrm{p} 125^{\mathrm{FAK}}$.

JNKs, named for their activity as Jun kinases are also known as stress induced kinase as they are activated in many cells by proinflammatory cytokines and environmental stress (Karin 1998). JNKs phosphorylate the amino terminal transactivation domain of the c-Jun transcription factor which can then dimerize with fos and control the expression of a number of genes. JNKs can be assayed using a GST-Jun amino terminal fusion protein either to bind and pull down active JNK followed by a kinase reaction or with an in gel kinase assay which has the advantage of separating

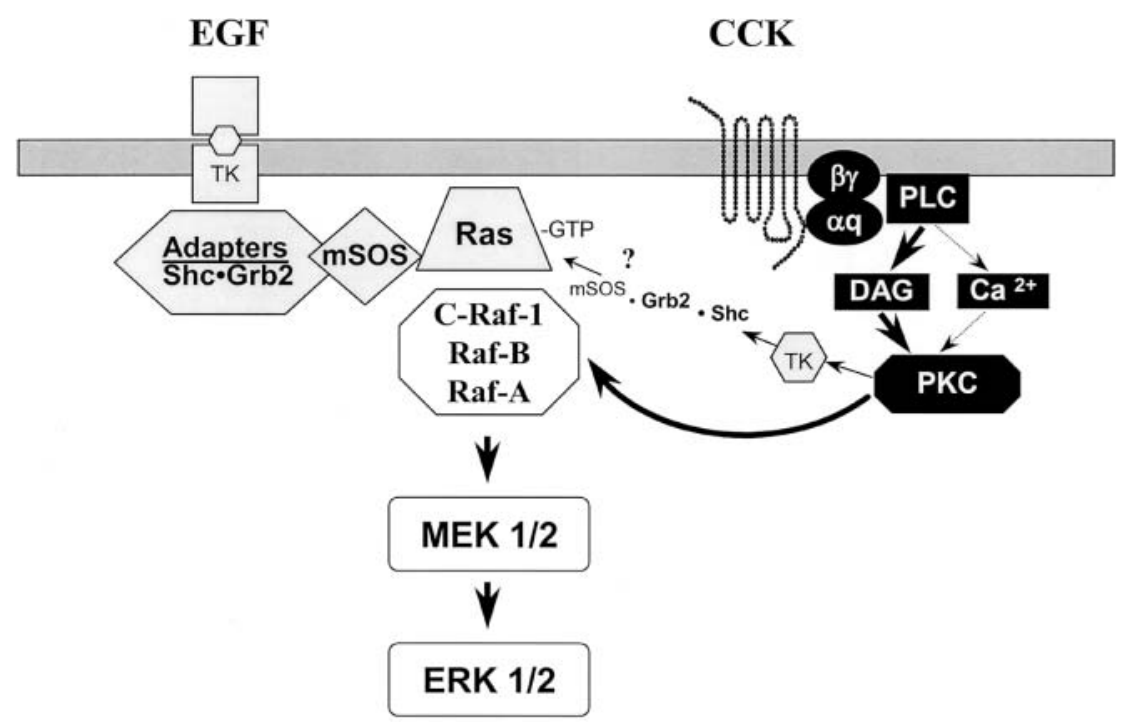

Fig. 2. Schematic depiction of the pathways leading to ERK $1 / 2$ activation stimulated by CCK and EGF receptors. While the EGF receptor signals by its tyrosine kinase (TK) activity through adaptor proteins to Ras, CCK has at most a minor potential pathway through Ras and a major pathway involving PKC acting to activate Raf. The multiple forms of Raf, MEK and ERKs listed have all been shown to be activated by CCK. Modified from Dabrowski et al. (1997). 
the p46 and p55 forms of JNK. Since JNK is activated similar to ERKs by dual phosphorylation, JNK activity can also be inferred by Western blotting with antibody to its specific phosphoepitope. The upstream kinases have not been studied in acinar cells but those active in other cells are listed in fig. 1. Dabrowski et al. (1996b) first showed that CCK could activate JNKs in pancreatic acini. Carbachol and bombesin had a smaller effect while VIP had no effect. When JNK and ERK activity were compared, the effect of CCK to activate JNKs was slower requiring 30 min. to reach maximum and also required a higher concentration of CCK. In vivo the activity of pancreatic JNK is quite low indicating that basal levels are increased by isolating acini. Caerulein infusion at physiological levels increased JNK 4 times while supraphysiological levels used to induce pancreatitis increased JNK activity 25 fold (Dabrowski et al. 1996b). The increase in JNK by high concentrations of CCK (or caerulein) may reprecent a stress response. At present, it is unknown whether JNK activation is protective or deleterious in pancreatitis and which genes are activated. A recent in vivo study showed that a JNK inhibitor blocked the oedema but not other components of pancreatitis (Wagner et al. 2000).

The p38 MAPKs were originally identified in yeast as a kinase activated by high osmolarity but are known to be activated by both stress and growth stimuli in mammalian cells. Four different isoforms are now known and designated $\alpha, \beta, \gamma$ and $\delta$. Their identified MAPKKs are known as MKK3, MKK4 and MKK6. At the level of small G proteins, Cdc42 is a known activator of this cascade (fig. 1). The presence of p38 MAPK in rat acinar cells was shown by Schäfer et al. (1998). CCK increased the activity of p38 measured using a GST-ATF2 fusion protein substrate after immunoprecipitation. p38 was activated rapidly within 1 min. and by physiological levels of CCK. Carbachol and bombesin but not VIP also activated p38, although the greatest increase was induced by hyperosmolarity. This was parallel by an increase in tyrosyl phosphorylation of the p38 activation site. Using a specific inhibitor of p38, SB203580, p38 was shown to be required for the activation of the downstream kinase, MAPKAP-kinase 2 in pancreatic acini. MAPKAPK-2 is known to phosphorylate HSP27 previously shown to be phosphorylated in response to CCK (Groblewski et al. 1997) and SB2030580 was found to block the phosphorylation of HSP27 induced by CCK (Schäfer et al. 1998). By use of a rhodamine phalloidin binding assay and confocal fluorescence microscopy it was shown that CCK induced changes in the actin cytoskeleton were largely blocked by SB203580. Thus, this pathway from p38 to HSP27 appears important for regulating the actin cytoskeyeton in acinar cells. That this effect was mediated by phosphorylation of HSP27 was confirmed by transiently over expressing HSP27 in CHO cells stably expressing CCKA receptors (Schäfer et al. 1999). The protection against CCK alterations in the actin cytoskeleton induced by HSP27 was lost when the phosphorylatable serine residues were mutated to glycine or alanine. p38 MAPK may also mediate some of the transcriptional regulation seen in response to

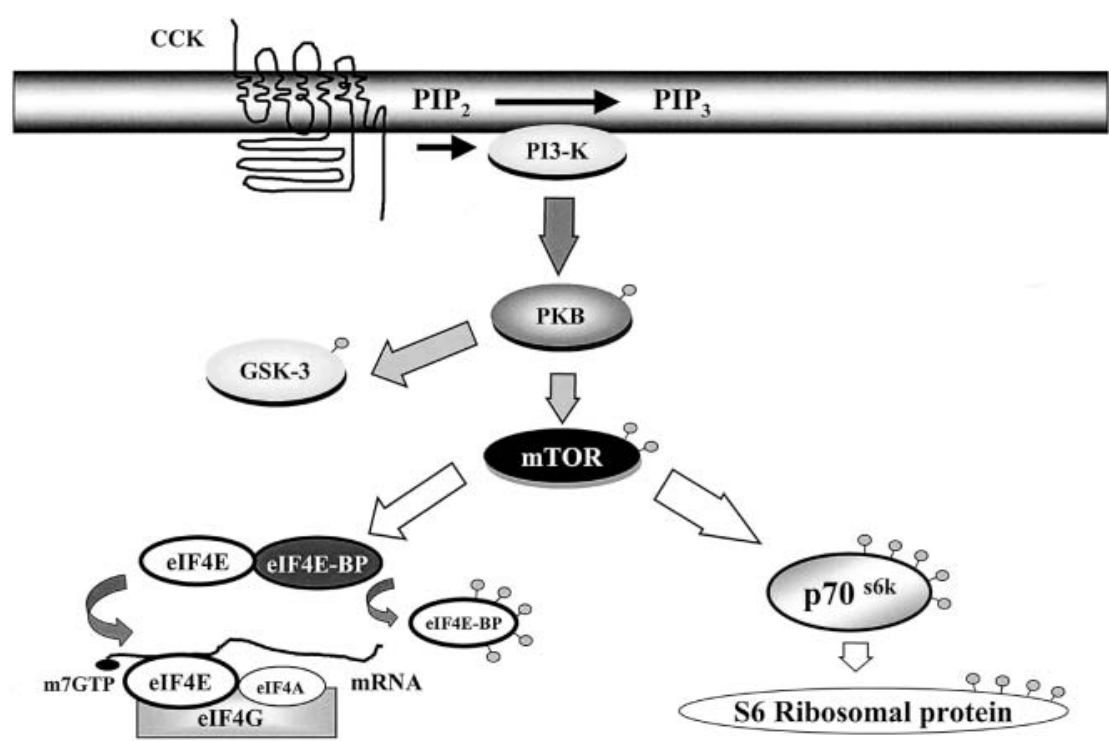

Fig. 3. CCK activates the PI3K-PKB-mTOR pathway to regulate translation initiation in pancreatic acinar cells. PI3K has actions both as a lipid kinase to phosphorylate inositol containing lipids and as a protein kinase where it phosphorylates and activates PKB which then phosphorylates and stimulates mTOR. PKB most likely has other actions such as phosphorylating GSK-3. mTOR is responsible (at least in part) for phosphorylating eIF4E-BP that results in release of eIF4E and the formation of the messenger RNA cap binding complex necessary for the global increase in transition. mTOR also phosphorylates p70 S6K which is responsible for phosphorylating ribosomal protein $\mathrm{S} 6$ which increases the translation of mRNAs with $5^{\prime}$ polypyrimide tracts. 
CCK as in other cells, p38 phosphorylates transcription factors including ATF-2, CHOP, and CREB.

\section{PI3K-PKB-mTOR signaling.}

A signaling pathway that primarily regulates protein synthesis but which also is required for mitogenesis and has anti-apoptotic and metabolic effects is the PI3K-PKBmTOR pathway. This pathway can be activated in acinar cells by CCK as well as by insulin and acetylcholine acting on $\mathrm{m} 3$ receptors. While the pathway includes three kinases in series similar to the MAP kinase cascades, each of the three has numerous downstream targets. Fig. 3 shows the pathway in a linear manner aimed at controlling protein synthesis but each of the three kinases has multiple downstream actions and their activation is more complicated than shown. Thus, PI3K as a protein kinase phosphorylates and activates protein kinase B also known as Akt while as a lipid kinase it increases the membrane content of 3 phosphorylated phosphoinositides and thereby activates other downstream proteins many of which bind to 3 phosphoinositides through pleckstrin homology domains (Cantrell 2001). Protein kinase B is a protein kinase with multiple targets including the mammalian Target of Rapamicin (mTOR) and glycogen synthase kinase-3(GSK-3) (Vanhaesebroeck \& Alessi 2000). Both PI3K and protein kinase B possess multiple isoforms and little is known regarding which isoform is present in acinar cells or as to how the CCK receptor activates PI3K. CCK increases the activity of protein kinase $\mathrm{B}$ in isolated rat acini (Sans \& Williams, unpublished results) while gastrin activates $\mathrm{PKB}$ in AR42J cells (Todisco et al. 2001).

More is known in acini of the targets of mTOR and how mTOR activation is related to the translational control of protein synthesis. Ribosomal protein S6 phosphorylation was one of the first regulatory phosphorylation events shown to be stimulated by CCK in acinar cells. The kinase phosphorylating S6 is now known to be p70 S6K and this kinase was shown to be phosphorylated and activated in response to CCK in isolated pancreatic acini (Bragado et al. 1997). Activation was blocked both by rapamycin, which binds to an intracellular receptor FKBP-12 and acts as an inhibitor of mTOR and by inhibitors of PI3K including Wortmannin and LY294002. Gastrin, acting on CCKB receptors also activates p70 S6K in AR42J cells (Seva et al. 1997). P70 S6K is activated by a hierarchical phosphorylation at multiple sites but phosphorylation by mTOR is critical. Antibodies directed at specific phosphoserine epitopes can also now be used to monitor p70 S6K activation. Phosphorylation of S6 is believed to facilitate translation of mRNA species containing a $5^{\prime}$ polypyrimidine tract (socalled TOP sequences) which are prominent in ribosomal proteins and translation factors. Thus, the acute translational upregulation of components of the translational machinery may increase the overall capacity of the cell for protein synthesis (Proud \& Denton 1997).

The other target of mTOR playing a role in translational control in acinar cells in response to $\mathrm{CCK}$ is the binding protein for eukaryotic initiation factor 4E (eIF4E-BP), also known as PHAS-I because it was identified as a heat and acid stable phosphoprotein regulated by insulin (Proud \& Denton 1997). This protein and a highly related second isoform are small proteins of $10-12 \mathrm{kDa}$ which are rapidly phosphorylated on both Ser and Thr residues in response to numerous external stimuli including hormones, cytokines, growth factors, amino acids and mitogens (Hershey \& Merrick 2000). At least 5 sites have been identified phosphorylated by multiple kinases but with mTOR the most important regulator. Phosphorylation of eIF4E-BP releases eIF4E, the mRNA cap binding protein which is probably the most rate limiting of the initiation factors. eIF4E then binds to a scaffolding protein eIF4G and eIF4A, a RNA helicase, to form a complex known as eIF4F which binds to the mRNA cap, commits this particular mRNA molecule to translation and leads to the binding of the 40S ribosomal preinitiation complex. CCK acts on isolated acini and in vivo to stimulate eIF4E-BP phosphorylation, the release of eIF4E and the formation of a eIF4E-eIF4G complex (Bragado et al. 1998 \& 2000). These actions are blocked by rapamycin and PI3K inhibitors all of which also blocked global protein synthesis in rat pancreatic acini, reducing basal synthesis and blocking the increase in response to CCK (Bragado et al. 1998). In AR42J cells, rapamycin similarly inhibits eIF4E-BP phosphorylation in response to gastrin (Pyronnet et al. 1998). Thus, both CCK receptors can activate this pathway.

In a separate signaling pathway CCK stimulation of acinar cells increases the phosphorylation of eIF4E itself (Bragado et al. 2000). This phosphorylation is known to enhance the affinity of eIF4E for the mRNA cap but the importance of this in regulating acinar cell protein synthesis is unclear. In other cells this phosphosylation has been shown to be mediated by mnk kinases which are activated by ERKs and p38 MAPK (fig. 1).

\section{Other signaling pathways.}

CCK stimulation of rat pancreatic acini and gastrin stimulation of AR42J cells is known to enhance the tyrosine phosphorylation of a variety of proteins (Williams 2001). The identity of some of these substrates as well as the specific tyrosine kinases involved are beginning to emerge. Several tyrosyl-phosphorylated proteins are adaptor proteins such as Shc and IRS-1 and others are present as part of the focal adhesion complex (p125 $5^{\mathrm{FAK}}$ and paxillin). Tyrosine kinases which have been reported to be activated by CCK include Src, p125 ${ }^{\mathrm{FAK}}$ and Pyk2. A potential role for some of these proteins is in regulation of the actin cytoskeleton. This almost certainly also involves the small $\mathrm{G}$ protein Rho.

Another signaling pathway recently shown to be activated by $\mathrm{CCK}$ in pancreatic acini is $\mathrm{NF}-\kappa \mathrm{B}$, a transcription factor important in the inflammatory response but with potential other roles. NF- $\kappa \mathrm{B}$ is sequestered in an inactive form in resting cells through an association with the inhibitory protein, I $\kappa \beta$. Cell stimulation leads to phosphorylation and degradation of IK $\beta$ followed by translocation of NF- $\kappa \beta$, to 
the nucleus where it regulates target genes. CCK was shown to activate $\mathrm{NF}-\kappa \beta$ through mechanisms involving increased cytoplasmic free calcium and activation of protein kinase $\mathrm{C}$ (Tando et al. 1999; Han \& Logsdon 2000). While activation of this pathway clearly occurs in caerulein-induced experimental pancreatitis, whether it has a physiological role in CCK action remains to be determined.

\section{Conclusion}

The last decade has shown that CCK receptors activate a multiplicity of intracellular signal transduction mechanisms in acinar cells that regulate not only secretion but also protein synthesis, growth and metabolism. These pathways are present in most differentiated cell types and the pathways activated by CCK in rodent acinar cells most likely occur in other target cells bearing CCK receptors. However, the cellular context often also affects the pathways activated and different cell types may contain different isoforms of signaling intermediates. Thus, although the acinar cell is an excellent model cell other cell types also need to be studied. In many cases the machanism by which the CCK receptor couples to a specific intracellular pathway is not fully understood. In some cases distinct heterotrimeric $\mathrm{G}$ proteins may be involved or the pathway is regulated by a classical second messenger such as $\mathrm{Ca}^{2+}$, cAMP or diacylglycerol (protein kinase $\mathrm{C}$ ). Crosstalk and interaction between signaling pathways is also an area for further investigation.

\section{References}

Bragado, M. J., A. Dabrowski, G. E. Groblewski \& J. A. Williams: CCK activates $\mathrm{p} 90^{\mathrm{rsk}}$ in rat pancreatic acini through protein kinase C. Amer. J. Physiol. 1997, 272, G401-G407.

Bragado, M. J., G. E. Groblewski \& J. A. Williams: p70 ${ }^{\mathrm{s} 6 \mathrm{k}}$ is activated by CCK in rat pancreatic acini. Amer. J. Physiol. 1997, 273, C101-C109.

Bragado, M. J., G. E. Groblewski \& J. A. Williams: Regulation of protein synthesis by cholecystokinin in rat pancreatic acini involves PHAS-1 and the p70 S6 kinase pathway. Gastroenterology 1998, 115, 733-742.

Bragado, M. J., M. Tashiro \& J. A. Williams: Regulation of the initiation of pancreatic digestive enzyme protein synthesis by cholecystokinin in rat pancreas in vivo. Gastroenterology 2000, 119, 1731-1739.

Cantrell, D. A.: Phosphoinositide 3-kinase signaling pathways. J. Cell Sci. 2001, 114, 1439-1445.

Chen, X., J. A. S. Edwards, C. D. Logsdon, S. A. Ernst \& J. A. Williams: Dominant negative Rab3D inhibits amylase release from mouse pancreatic acini. J. Biol. Chem. 2002, 277, 1800218009.

Dabrowski, A., G. E. Groblewski, C. Schäfer, K.-L. Guan \& J. A Williams: Cholecystokinin and EGF activate a MAPK cascade by different mechanisms in rat pancreatic acinar cells. Amer. J. Physiol. 1997a, 273, C1472-C1479.

Dabrowski, A., K. M. Detjen, C. D. Logsdon \& J. A. Williams: Stimulation of both CCK-A and CCK-B receptors activates MAP kinases in AR42J and receptor-transfected $\mathrm{CHO}$ cells. $\mathrm{Di}$ gestion 1997b, 58, 361-367.

Dabrowski, A., J. A. VanderKuur, C. Carter-Su \& J. A. Williams: Cholecystokinin stimulates formation of Shc-Grb2 complex in rat pancreatic acinar cells through a protein kinase C-dependent mechanism. J. Biol. Chem. 1996a, 271, 27125-27129.

Dabrowski, A., T. Grady, C. D. Logsdon \& J. A. Williams: Jun kinases are rapidly activated by cholecystokinin in rat pancreas both in vitro and in vivo. J. Biol. Chem. 1996b, 271, 5686-5690.

Duan, R.-D. \& J. A. Williams: Cholecystokinin rapidly activates mitogen-activated protein kinase in rat pancreatic acini. Amer. J. Physiol. 1994, 267, G404-G408.

Duan, R.-D., C.-F. Zheng, K.-L. Guan \& J. A. Williams: Activation of MAP kinase kinase (MEK) and Ras by cholecystokinin in rat pancreatic acini. Amer. J. Physiol. 1995, 268, G1060-G1065.

Enslen, H., J. Raingeaud \& R. J. Davis: Selective activation of p38 mitogen-activated protein (MAP) kinase isoforms by the MAP kinase kinases MKK3 and MKK6. J. Biol. Chem. 1998, 273, 1741-1748.

Groblewski, G. E., T. Grady, N. Mehta, H. Lambert, C. D. Logsdon, J. Landry \& J. A. Williams: Cholecystokinin stimulates heat shock protein 27 phosphorylation in rat pancreas both in vivo and in vitro. Gastroenterology 1997, 112, 1354-1361.

Han, B. \& C. D. Logsdon: CCK stimulates mob-1 expression and NF- $\kappa \mathrm{B}$ activation via protein kinase $\mathrm{C}$ and intracellular $\mathrm{Ca}^{2+}$ Amer. J. Physiol. 2000, 278, C344-C351.

Hershey, J. W. \& W. C. Merrick: Pathway and mechanism of initiation of protein synthesis. In: Translational control of gene expression. Ed.: M. Matthews. Cold Spring Harbor, New York, Cold Spring Harbor Laboratory Press 2000, 33-88.

Karin, M.: Mitogen-activated protein kinase cascades as regulators of stress responses. Ann. N. Y. Acad. Sci. 1998, 851, 139-146 (Review).

Liddle, R. A., I. D. Goldfine \& J. A. Williams: Bioassay of plasma cholecystokinin in rats: effects of food, trypsin inhibitor, and alcohol. Gastroenterology 1984, 87, 542-549.

Matozaki, T. \& J. A. Williams: Multiple sources of 1,2-diacylglycerol in isolated rat pancreatic acini stimulated by cholecystokinin J. Biol. Chem. 1989, 264, 14729-14734.

Nicke, B., M. J. Tseng, M. Fenrich \& C. D. Logsdon: Adenovirusmediates gene transfer of RasN17 inhibits specific CCK actions on pancreatic acinar cells. Amer. J. Physiol. 1999, 276, G499G506.

Petersen, O. H., D. Burdakov \& A. V. Tepikin: Polarity in intracellular calcium signaling. BioEssays 1999, 21, 851-860.

Proud, C. G. \& R. M. Denton: Molecular mechanisms for the control of translation by insulin. Biochem J. 1997, 328, 329-341.

Pyronnet, S., A.-C. Gingras, M. Bouisson, A. Kowalski-Chauvel \& C. Seva: Gastrin induces phosphorylation of eIF4E binding protein 1 and translation initiation of ornithine decarboxylase mRNA. Oncogene 2000, 16, 2219-2227.

Schäfer, C. \& J. A. Williams: Stress kinases and heat shock proteins in the pancreas: possible roles in normal function and disease. $J$. Gastroenterol. 2000, 35, 1-9.

Schäfer, C., P. Clapp, M. J. Welsh, R. Benndorf \& J. A. Williams: HSP27 expression regulates CCK-induced changes of the actin cytoskeleton in CHO-CCK-A cells. Amer. J. Physiol. 1999, 277, C1032-C1043.

Schäfer, C., S. E. Ross, M. J. Bragado, G. E. Groblewski, S. A Ernst \& J. A. Williams: A role for the p38 mitogen-activated protein kinase/Hsp 27 pathway in cholecystokinin-induced changes in the actin cytoskeleton in rat pancreatic acini. J. Biol. Chem. 1998, 273, 24173-24180.

Seva, C., A. Kowalski-Chauvel, L. Daulhac, C. Barthez, N. Vaysse \& L. Pradayrol: Wortmannin-sensitive activation of p70S6-kinase and MAP-Kinase by the G protein-coupled receptor, G/ CCK $_{\mathrm{B}}$. Biochem. Biophys. Res. Commun. 1997, 238, 202-206.

Tando, Y., H. Algul, M. Wagner, H. Weidenbach \& G. Adler: Caerulein-induced NF- $\mathrm{NB} /$ Rel activation requires both $\mathrm{Ca}^{2+}$ and protein kinase C as messengers. Amer. J. Physiol. 1999, 277, G678-G686.

Todisco, A., S. Ramamoorthy, T. Withan, N. Pausawasdi, S. Srinivasan, C. J. Dickinson, F. K. Askara \& D. Krametter: Molecular 
mechanisms for the antiapoptotic action of gastrin. Amer. J. Physiol. 2001, 280, G298-G307.

Vanhaesebroeck, B. \& D. R. Alessi: The PI3K-PDK1 connection: more than just a road to PKB. Biochem. J. 2000, 346, 561-576.

Wagner, A. C. C., L. Mazzucchelli, M. Miller, A. M. Camoratto \& B. Göke: CEP-1347 inhibits caerulein-induced rat pancreatic JNK activation and ameliorates caerulein pancreatitis. Amer. J. Physiol. 2000, 278, G165-G172.

Widmann, C., S. Gibson, M. B. Jarpe \& G. L. Johnson: Mitogenactivated protein kinase: conservation of a three-kinase module from yeast to human. Physiol. Rev. 1999, 79, 143-180.

Williams, J. A., M. Korc \& R. L. Dormer: Action of secretagogues on a new preparation of functionally intact, isolated pancreatic acini. Amer. J. Physiol. 1978, 235, E517-E524.
Williams, J. A.: Intracellular signaling mechanisms activated by cholecystokinin-regulating synthesis and secretion of digestive enzymes in pancreatic acinar cells. Annu. Rev. Physiol. 2001, 63, 77-97.

Williams, J. A.: Receptor biology and intracellular regulatory mechanisms in pancreatic acinar cells. Current Opinion Gastroenterol. 2002, 18, 529-535.

Williams, J. A. \& G. T. Blevins: Cholecystokinin and regulation of pancreatic acinar cell function. Physiol. Rev. 1993, 73, 701-721.

Yule, D. I. \& J. A. Williams: Stimulus-secretion coupling in the pancreatic acinus. In: Physiology of the gastrointestinal tract, 3rd Edition. Ed.: L. R. Johnson. Raven Press, New York, 1994, pp. $1447-1472$ 